\title{
Mechanisms of Inhibitory Effects of Polysubstituted Pyrimidines on Prostaglandin E2 Production ${ }^{+}$
}

\author{
Zdeněk Zídek ${ }^{1, *}$, Viktor Kolman ${ }^{2}$, Ondřej Baszczyňski ${ }^{2}$, Filip Kalčic ${ }^{2}$, Daniel Board ${ }^{2}$ \\ and Zlatko Janeba ${ }^{2}$ \\ 1 Institute of Experimental Medicine, The Czech Academy of Sciences, 14220 Prague, Czech Republic \\ 2 Institute of Organic Chemistry and Biochemistry, The Czech Academy of Sciences, \\ 16610 Prague, Czech Republic \\ * Correspondence: zdenek.zidek@iem.cas.cz \\ + Presented at the 2nd Molecules Medicinal Chemistry Symposium (MMCS): Facing Novel Challenges in \\ Drug Discovery, Barcelona, Spain, 15-17 May 2019.
}

Received: 7 August 2019

Keywords: pyrimidine analogues; prostaglandin E2; prostaglandin E2 synthase-1 inhibitors; anti-inflammatory activity

The pyrimidine heterocycle represents an elemental structural motif of numerous drugs. We have synthesized a large series of original derivatives possessing different substituents at C-2, C-4, C-5, and C-6 positions of the pyrimidine ring. The vast majority of prepared pyrimidines inhibit prostaglandin $\mathrm{E}_{2}\left(\mathrm{PGE}_{2}\right)$ production as revealed in vitro in the lipopolysaccharide (LPS)-stimulated mouse macrophages [1,2]. A number of them are effective at sub-micromolar concentration. The compounds are devoid of cytotoxic effects. They do not inhibit activities of phospholipase A2 (sPLA2), cyclooxygenases COX-1 and COX-2, and important enzymes in the PGE2 biosynthesis pathway. A plausible explanation for the mechanism of PGE2-inhibitory effects of pyrimidines is provided by findings showing substantial inhibition of activity of the terminal enzyme in PGE2 formation, i.e., microsomal prostaglandin E2 synthase-1 (mPGES-1). The IC $C_{50}$ characterizing the potential of compounds to reduce mPGES-1 activity on one site and LPS-induced PGE2 production on the other one are statistically significantly correlated.

Pyrimidine derivatives exhibit anti-inflammatory activity in vivo, as demonstrated by the significant reduction of carrageenan-induced paw oedema in rats. The findings suggest that pyrimidine inhibitors of mPGES- 1 activity and consequent PGE2 $_{2}$ production may be considered as promising candidates for further preclinical research and development of novel non-steroidal anti-inflammatory drugs.

Acknowledgments: The research was supported by grant TE01020028 from the Technology Agency of the Czech Republic.

\section{References}

1 Zídek, Z.; Kverka, M.; Dusilová, A.; Kmoníčková, E.; Jansa, P. Dual inhibition of nitric oxide and prostaglandin E2 production by polysubstituted 2-aminopyrimidines. Nitric Oxide 2016, 57, 48-56.

2 Kolman, V.; Kalčic, F.; Jansa, P.; Zídek, Z.; Janeba, Z. Influence of the C-5 substitution in polysubstituted pyrimidines on inhibition of prostaglandin E2 production. Eur. J. Med. Chem. 2018, 156, 295-301. 